\title{
CHANGING DIMENSION IN AN AUTONOMOUS ODE WHEN TRANSFORMED IN A FODE
}

\author{
BERENICE C. DAMASCENO ${ }^{1}$, CÉLIA A. DOS REIS ${ }^{1}$, LUCIANO BARBANTI ${ }^{1}$.
}

1. Departamento de Matemática, Faculdade de Engenharia, UNESP campus de Ilha Solteira

Al. Rio de Janeiro, 266 - 15385-000 - Ilha Solteira - SP.

E-mails: berenice@mat.feis.unesp.br; celia@mat.feis.unesp.br;

barbanti@mat.feis.unesp.br

\begin{abstract}
In this work we will showing that it is possible to choose the dimension of the phase plane of an autonomous Ordinary Differential Equation (ODE) when we consider fractional derivatives through a system of Fractional Ordinary Differential Equation (FODE). This affects the classical structure of the switching-locus in the phase space of the system.
\end{abstract}

Keywords— Minimal time, control, fractional calculus, FODE.

\section{Introduction}

Nowadays we have in the literature a huge amount of results having as object the Fractional Ordinary Differential Equations (FODE). This is due mainly because the fractional equations turns possible the consideration of a more complex structure than the classical one (with integer order derivatives) allowing in this way a more accurate point of view in the applications. This aspect can be seen in Benson (2000), Caputo (1967), Näsholm (2013) and Chen (2010).

Due to the novelty of the field we have always present- when developing arguments on FODE- the necessity in to be comparing results with the ones coming from the classical field of the ODE.

Our work is inserted in this point of view.

We are proposing here a new substitution of variables in a OD transforming in this way the ODE in a general FODE .We study as one example- with the purpose in to be demonstrating the method-the phase portrait concerning the classical example in control theory of the transference of a rocket car from an initial position and velocity to the total rest in minimal time by means of bounded controls.

\section{Fractional derivative}

In fractional calculus we have several definitions for the calculation of the fractional order derivative for a real function. The two most commonly used are the Liouville-Riemann or the Caputo notions of fractional derivative. The Caputo fractional derivative has been used with better results in FODE because differently from other definitions of fractional derivative, it permits to take the initial conditions in the equation in the same manner as for ODE. This can be seen in Rida (2011).

We use throughout this paper as definition of fractional order derivative the Caputo one:

Definition 1: Let be

$$
\begin{gathered}
x: \mathbb{R} \rightarrow \mathbb{R} \text {, and } m-1<a \leq m, \\
\qquad m \in \mathbb{N}
\end{gathered}
$$

We say that the fractional derivative of

$$
x(t),(t>0)
$$

in the sense of Caputo is:

$$
D^{a} x(t)=\frac{1}{\Gamma(m-a)} \int_{0}^{t}(t-s)^{m-a+1} x^{(m)}(s) d s
$$

where $\Gamma$ is the symbol for the usual gamma function. According to the definition above we have:

the class

$$
\left\{D^{\alpha} ; \alpha>0\right\}
$$

obeys the semi-group property

$$
D^{\alpha}\left(D^{\beta} x\right)=D^{\alpha+\beta} x
$$

Further, if

$$
x(t)=t^{n}, \quad n \in \mathbb{N}
$$

then

$$
D^{a} x(t)=\frac{\Gamma(n+1)}{\Gamma(n-a+1)} t^{n-a}
$$

and if

$$
x(t)=\text { constant }
$$

then

$$
D^{a} x(t)=0
$$

In the next section we introduce the problem which will be the support for the exhibition of our results. 


\section{The rocket car. The classical ODE case}

The rocket car problem as known in the literature on controlled ODE consists in to take an object with unitary mass subjected to a force $F$ bounded by the constraint

$$
|F(t)| \leq 1
$$

The movement obeys the second order equation with $x: R \rightarrow R$

$$
\ddot{x}=F(t)
$$

If we use

$$
(x(t), y(t))
$$

the position and velocity ,in the vector state coordinates [ with value

$$
\left(x_{0}, y_{0}\right)
$$

at

$$
t=0,]
$$

We get the equivalent in the plane:

$$
\begin{aligned}
& \dot{x}=y(t) \\
& \dot{y}=F(t)
\end{aligned}
$$

The control problem consists in to drive the car from the position

$$
\left(x_{0}, y_{0}\right)
$$

to

in minimal time along the

$$
\ddot{x}=F(t)
$$

orbits.

According to the bang-bang principle - see Macki (1989) -we know that if there is a force $F$ performing such transference, then there exists a mapping $F_{0}$ which realizes the transference in minimal time and $F_{0}$ satisfies

$$
\left|F_{0}(t)\right|=1
$$

Moreover $F_{0}$ presents at most one jump from the value 1 to the value -1 (or vice versa).In this way we have two essential systems in the plane when considering this kind of transference:

$$
\dot{x}=y(t), \dot{y}=1
$$

and

$$
\dot{x}=y(t), \dot{y}=-1 \text {. }
$$

In a very simple way we have for the two systems, respectively:

$$
\begin{gathered}
y(t)=t+c, \\
x(t)=\frac{t^{2}}{2}+c t+d
\end{gathered}
$$

And

$$
y(t)=t+c
$$

$$
x(t)=-\frac{t^{2}}{2}+c t+d .
$$

In this way we are able in to find the following relation between $\mathrm{x}$ and $\mathrm{y}$ in the phase plane

and

$$
y(t)^{2}-y_{0}^{2}=2\left(x(t)-x_{0}\right)
$$

$$
y(t)^{2}-y_{0}^{2}=-2\left(x(t)-x_{0}\right)
$$

Having these two relations in mind we can easily determine in the phase plane for

$$
\ddot{x}=F(t),
$$

the strategy used to be driving

$$
\left(x_{0}, y_{0}\right) \text { to }(0,0) \text {. }
$$
fact)

Call $\varphi_{+}$(respectively: $\varphi_{-}$) the curve (an orbit in

$$
y(t)^{2}=2 x(t)
$$

of the first system in the region

$$
x \geq 0, y \leq 0
$$

(respectively :

$$
y(t)^{2}=-2 x(t)
$$

of the second system in the region $x \leq 0, y \geq 0$ ).

The curve

$$
\varphi=\varphi_{-} \cup \varphi_{+}
$$

splits the plane $(x, y)$ in two parts and $(0,0) \in \varphi$.If $\left(x_{0}, y_{0}\right)$ is on the superior part with relation to $\varphi$ we must apply the control -1 and drive the point according the second system until to reach the orbit $\varphi_{+}$and 
finally reach $(0,0)$ by using the first system (controlled by 1$)$.If by other hand, $\left(x_{0}, y_{0}\right)$ is on the inferior part then we act in a symmetric manner.

\section{Transforming the ODE in a general FODE}

As a general task, the equation $\ddot{x}=F(t)$, could be spliced in

$$
D^{a}\left(D^{2-a}\right)=F(t)
$$

with

$$
0<a \leq 1
$$

Then by constructing the correspondent systems as above, in the present case we get the systems named here $\left(\mathrm{O}^{+}\right)$and $(\mathrm{O}-)$ : the $\left(\mathrm{O}^{+}\right)$is

$$
D^{2-a} x=y, D^{a} y=1,
$$

and the (O-) one is:

$$
D^{2-a} x=y, D^{a} y=-1 .
$$
$(\mathrm{O}+)$

According the Caputo derivative we have in

$$
y(t)=\frac{t^{a}}{\Gamma(1+a)}+c, x(t)=A t^{2}+B t^{a}+C
$$

showing in this way that for each $0<a \leq 1$ we will get another geometric configurations in the phase portrait when solving the problem of the rocket car as stated above.

\section{Increasing the dimension in the rocket car problem}

The fractional derivatives open a possibility that we do not have in the case in which we are using the integer derivatives. By using fractional derivatives we can increase the dimension in the decomposition of an autonomous ODE. Consider the case of the rocket car, again.

If we are using only integer derivatives, the equation

$$
D^{2} x=\ddot{x}=1
$$

can be decomposed into lower order derivatives in this way only

$$
D^{1}\left(D^{1} x\right)=1 .
$$

However, if we are making use of general fractional derivatives we can decompose the rocket car equation $\mathrm{n}$ times in another way

$$
D^{a_{1}} D^{a_{2}} \ldots . D^{a_{n}} x=1,
$$

with

$$
a_{1}+a_{2}+\cdots+a_{n}=2 .
$$

A little bit calculation shows that we get

$$
\begin{gathered}
x=A_{1} t^{\left(\sum_{i=1}^{n} a_{i}\right)}+A_{2} t^{\left(\sum_{i=2}^{n} a_{i}\right)}+A_{3} t^{\left(\sum_{i=3}^{n} a_{i}\right)}+\cdots \\
+A_{n} t^{a_{n}}+A
\end{gathered}
$$

where $A_{i}$ and $A$ are constants determined in the computation of the Caputo derivative for a polynomial. Making

$$
\begin{gathered}
D^{a_{n} x}=x_{n-1}, \\
D^{a_{n-1}} x_{n-1}=x_{n-2}, \\
\cdots \\
D^{a_{2}} x_{2}=x_{1}, \\
D^{a_{1}} x_{1}=1,
\end{gathered}
$$

we have a n dimensional system, equivalent to

$$
D^{1}\left(D^{1} x\right)=1 .
$$

This kind of division enable us to make changes in the usual geometry in the synthesis of the switching-locus for the minimal time control when we drive the rocket car from a state to the origin.

\section{Contribution of this article}

Along with the possibility in to be choosing the dimension of the phase plane of an autonomous Ordinary Differential Equation (ODE) when we consider fractional derivatives in a system of Fractional Ordinary Differential Equation (FODE) we are having the opportunity in to be transforming the usual geometry of the switching-locus design in the phase-plane in another one. This affects the procedure in to be taking decisions on the optimality when we need to be applying a sequence of controls.

Notice that all these aspects are being discussed in a work in preparation by the authors, see Barbanti (2013).

\section{References}

Benson, D., Wheatcraft, S., Meerschaert, M., (2000). "Application of a fractional advection-dispersion equation." Water Resources Res 36, 1403-1412. DOI: 10.1029/2000WR900031

Huang, H. S. and Lu, C. N (1994). Efficient Storage Scheme and Algorithms for W-matrix Vector Multiplication on Vector Computers. IEEE Transactions on Power Systems, Vol.9, No. 2; pp. 1083- 1094. DOI: $10.1109 / 59.317622$ 
Caputo M. (1967). "Linear model of dissipation whose Q is almost frequency independent-II". Geophys. J. R. Astr. Soc. 13: 529-539. DOI:

10.1111/j.1365-246X.1967.tb02303.x

Näsholm, S.P., and Holm, S. (2013). "On a Fraction-

al Zener Elastic Wave Equation," Fract. Calc. Appl. Anal. Vol. 16, No 1 pp. 26-50, DOI: 10.2478 .

Chen, W., Sun, H.G., Zhang, X., Korosak, D., (2010). "Anomalous diffusion modeling by fractal and fractional derivatives." Computers and Mathematics with Applications, 59(5), 1754-1758. DOI: 10.1016/j.camwa.2009.08.020

Rida, S.Z., Arafa, A.A.M.,(2011). New method for solving linear fractional differential equations. Int. J. of Dif. Eq. Doi 1011255.

Macki, J., Strauss, A. (1989). Introduction to optimal control theory,Springer Verlag (NY).

Barbanti, L. Damasceno, B.C., Reis .A, (2013). Optimal control theory for FODE (to appear-soon). 